\title{
Molecular Dynamics Simulations of Laser induced Ablation for Micro Propulsion
}

\author{
Daniel J. Förster, Stefan Scharring, Johannes Roth, Hans-Albert Eckel
}

\begin{abstract}
A new concept of micro propulsion based upon laser ablation MICROLAS was introduced by the Institute of Technical Physics (ITP) of DLR Stuttgart. Pulsed lasers are used for material removal of a target. The amount of removed material should be variable due to the tunability of input laser energy and repetition rate, resulting in well defined impulse bits and low small thrusts down to the sub- $\mu \mathrm{N}$ scale. We present a modeling approach of laser ablation in order to calculate important figures of merit in aerospace engineering. The program applied is IMD [25, 18] (http://imd.itap.uni-stuttgart.de), an open source molecular dynamics package of the Institute of Functional Materials Quantum Technologies (FMQ). Results are compared with a hydrodynamic code, VLL (http: //vll.ihed.ras.ru), as well as with experimental investigations.
\end{abstract}

Daniel J. Förster

Institut für Funktionelle Materialien und Quantentechnologien, Universität Stuttgart, email: foerster@itap.physik.uni-stuttgart.de

Stefan Scharring

Institut für technische Physik, Deutsches Zentrum für Luft- und Raumfahrttechnik, e-mail: stefan.scharring@dlr.de

Johannes Roth

Institut für Funktionelle Materialien und Quantentechnologien, Universität Stuttgart, email: johannes@itap.physik.uni-stuttgart.de

Hans-Albert Eckel

Institut für technische Physik, Deutsches Zentrum für Luft- und Raumfahrttechnik, e-mail: hans-albert.eckel@dlr.de 


\section{Introduction}

Very precise position control systems are needed in order to fulfill requirements of projects in earth and space exploration planned in past years. Existing thruster concepts have problems of endurable precise regulation of thrusts down to a few $\mu \mathrm{N}$, needed for accurate compensation of forces acting on satellites. Examples are chemical engines like hydrazine boosters, pulsed plasma thrusters or arcjets. An alternative to existing concepts in $\mathrm{nN}-\mu \mathrm{N}$ regime like nano-FEEPs, MEMS-ion thrusters or HEMP thrusters was introduced by the ITP [1]. It is based upon laser ablation of a (solid) propellant material and in the future shall guarantee position control of pretentiously missions like LISA Pathfinder [2] or MICROSCOPE [4]. At the FMQ ultrashort pulses in the fs-regime have been investigated through simulations. An existing Molecular Dynamics code of the institute, IMD, was under further development to enable the simulation of pulsed laser ablation [26]. This report investigates on the validation of IMD for calculations in the aerospace field. At the DLR facility in Stuttgart, laser ablation experiments are develop. Partially, the measurement of important quantities such as impulse coupling coefficient, specific impulse and angle distributions of neutral and charged particles were performed. Data of these will be compared with simulations.

The general concept of laser-ablation micropropulsion was given by Phipps who introduced a thruster concept using ms-laser pulses [15]. Later developments used ns-laser pulses [15]. MICROLAS is a new propulsion concept of a fine tuneable and controlable thruster in the $\mu \mathrm{N}$-regime. The intended structure is sketched in figure 1. Main and probably heaviest component is a laser source which beam propagates through a system of lenses and mirrors without any moving mechanical parts. This new concept is called inertia-free since the only impulse change comes from the ablation process itself. This occurs when the laser beam hits the propellant plate. In experiments at DLR mainly the laser-matter interaction has been analyzed until now in the laboratory.

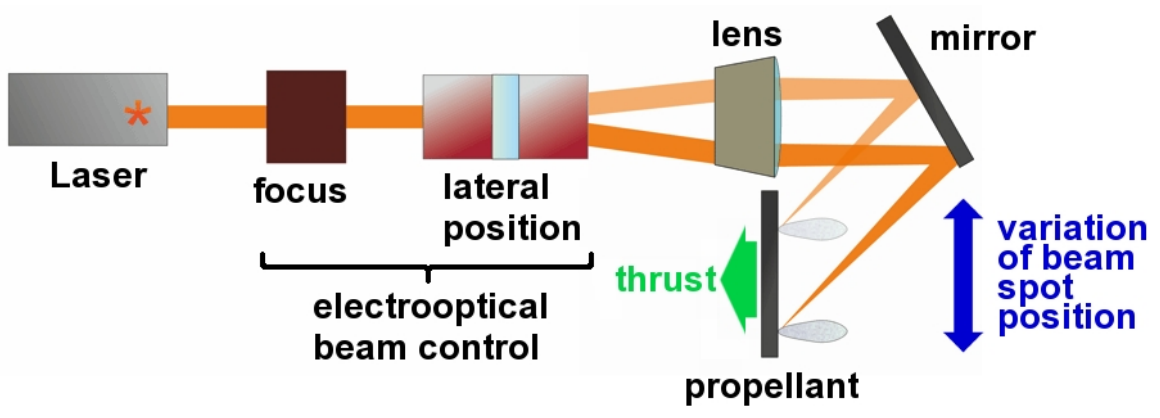

Fig. 1: Concept sketch of MICROLAS propulsion, adapted from [10] 
Main interests in simulations on atomistic scales, on one hand, come from the ablation process itself. The question is what processes in the microscopic world have a direct influence on macroscopic values. How are domains on the surface and within the material reorganized when a laser beam of high energy hits the target? Where does the energy go to? Does overlap of a beam spot with an already dug hole has an influence? What are the ratios of matter states and does ionization play a big role? In the long run, the evolution of the velocity and mass distribution in time and space is of big interest. The amount of ablated material and its potential of contaminating the propulsion system itself plays a big role when looking at main mission parameters like lifetime and possible time of operation. But the relapse of the material is also important in missions where the MICROLAS propulsion is designed for. Dirty lenses or current conducting shells may induce a variety of problems.

\section{Modeling}

\subsection{Molecular Dynamics and the IMD code}

In Molecular Dynamics (MD) simulations particles interact with each other under the influence of a potential $V(r)$. The interactions used in this investigation were the Embedded Atom Method-potentials of Ercolessi and Adams for aluminum [5] which are well established for metals. Newton's equations of motion are solved by integrators, in IMD a leapfrog integrator is used [26]. At each simulation step $t \rightarrow t+\Delta t$ the forces are calculated from the interaction potentials by the negative gradient

$$
\boldsymbol{F}=-\nabla V(\boldsymbol{r})=-\nabla V(r)
$$

where the second part comes from the assumption that the interactions just exhibit a proportionality to the distance. With the forces the coordinates are updated to new positions.

\subsection{Laser-matter interaction}

When energy is coupled into a sample by laser photons, the electrons react by gaining energy. The energy transport over the sample is no longer just driven by collisions of atoms as in equilibrium but by quantum mechanical effects. Since it is not directly possible in MD simulations to consider quantum effects yet, a macroscopic model was implemented to describe the heat transport within metallic samples properly. The two temperature model (TTM) describes the evolution of heat with three measurable main parameters in a 
set of nonlinear differential equations [26]:

$$
\begin{gathered}
C_{e}\left(T_{e}\right) \frac{\partial T_{e}}{\partial t}=\nabla\left[K_{e} \nabla T_{e}\right]-G\left(T_{e}-T_{l}\right) \\
C_{l}\left(T_{l}\right) \frac{\partial T_{l}}{\partial t}=\nabla\left[K_{l} \nabla T_{l}\right]+G\left(T_{e}-T_{l}\right)
\end{gathered}
$$

The indices $e$ and $l$ here stand for electrons and ions. The heat transfer from the electron subsystem to the lattice is described by the term $\left(T_{e}-T_{l}\right)$, with $G$ being the electron-phonon coupling constant. $C_{e, l}$ are heat capacities of the subsystems, $K_{e, l}$ are heat conductivities. The laser source term can be coupled to the heat equations via the electronic system via a laser power density term $S(\boldsymbol{r}, t)$. The phenomenological TTM equations describe heat transport in continuum, so usually they are solved by finite difference schemes (FD). Since we simulate on an atomistic level, just the electronic heat transport is described in continuum as in equation (2). The ionic movement is of course influenced by the behavior of the electrons, but their positions directly refer to atomic coordinates in the MD simulation. A hybrid simulation model was implemented in IMD in the following way [8]:

$$
\begin{aligned}
C_{e}\left(T_{e}\right) \frac{\partial T_{e}}{\partial t} & =\nabla\left[K_{e} \nabla T_{e}\right]-G\left(T_{e}-T_{l}\right)+S(\boldsymbol{r}, t) & & \text { FD } \\
m_{i} \frac{\partial^{2} \boldsymbol{r}_{i}}{\partial t^{2}} & =\boldsymbol{F}_{i}+\xi m_{i} \boldsymbol{v}_{i}^{T} & & \text { MD } \\
\xi & =\frac{\frac{1}{n} \sum_{k=1}^{n} G V_{N}\left(T_{e, k}-T_{l}\right)}{\sum_{j} m_{j}\left(v_{j}^{T}\right)^{2}} & & \text { coupling }
\end{aligned}
$$

$m_{i}, \boldsymbol{r}_{i}$ and $\boldsymbol{F}_{i}$ correspond to the MD system, the index $i$ stands for an atom, the forces $\boldsymbol{F}_{i}$ come from the interatomic interactions. The ions and electrons are calculated within two different systems, one belonging to MD space, the other to FD space, in which the electron heat transport is calculated. Here, the so called thermal velocity $v_{i}^{T}=v_{i}-v_{\text {com }}$ is introduced, where $v_{\text {com }}$ is the center of mass velocity of atoms in a FD cell. The two systems are coupled via $\xi$. For further details of the model see [24, 26].

The simulation results obtained with the interactions implemented in IMD show good agreements with experimental results in the fs-laser pulse regime $[24,26]$. However, there are limitations. A particle based algorithm that takes quantum effects directly into account would perfectly describe the atomistic world. Currently, however, all material parameters needed for the model have to be extracted from experiments. Most parameters are defined as constant at present, although in principle all are at least temperature dependent: 


$$
\begin{array}{ll}
\text { electron-phonon coupling parameter } & G(T):=G \\
\text { heat capacity } & C(T):=\gamma \cdot T \\
\text { thermal conductivity } & K(T):=K \\
\text { reflectivity } & R(T, \lambda, P):=R
\end{array}
$$

First principle calculations show that even the behavior of simple metals differs from these assumptions under non-equilibrium conditions [12]. Deviations occur for aluminum, copper and gold in heat conductivity $C$ and the electron-phonon coupling factor $G$. For $C$, a simple relation is already implemented, assuming a linear behavior. Here deviations lie in the range of decades, so the linear approximation covers the effect partially although the shape dependence is not completely correct. $K$ and $G$ deviate for aluminum at the same magnitude as the values themselves, so the constant approximation represents measured effects quite well. Reflectivity, however, in general is dependent of at least three physical values being temperature $T$, wavelength $\lambda$ and polarization $P$. The general laser source term applied exhibits the temporal shape of a Gaussian pulse:

$$
S(x, y, z, t)=S_{0} e^{-\frac{1}{2} \frac{\left(t-t_{0}\right)^{2}}{\sigma_{t}^{2}}} I(y, z) e^{-\mu x} \quad .
$$

Here $\sigma_{t}$ is the time at which $S(\boldsymbol{r}, t)$ decreased to a value of $1 / \sqrt{e} S_{0}, t_{0}$ is the time of the peak maximum. In x-direction the applied laser energy is decaying via the Lambert-Beer's law with $\mu$ being the inverse absorption length, $[\mu]=m^{-1}$, at which the intensity in x-direction decreases to $1 / e S_{0}$. The parameters used in simulations can be connected directly with common laser parameters used in experiments. By integration over time and space the total laser energy is provided via

$$
E=\frac{(2 \pi)^{3 / 2} S_{3 D} \sigma_{t} \omega_{0}^{2}}{(1-R) \mu}
$$

By integration over time and just the $\mathrm{x}$-coordinate the fluence

$$
\Phi=\frac{\sqrt{2 \pi} S_{1 D} \sigma_{t}}{(1-R) \mu}
$$

can be derived. The reflectivity $R$ is a unitless percentual constant that is fixed and is introduced during integration via

$$
E \cdot(1-R)=\int S(\boldsymbol{r}, t)
$$


The reflectivity $R$ is treated as conversion parameter via

$$
\Phi=\frac{\sigma_{e}}{(1-R)}
$$

since in IMD the quantity used to define the applied energy is the fluence parameter $\sigma_{e}$.

\subsection{Calculation of aerospace parameters}

Most microthruster systems for space applications are characterized by two main parameters, the so called specific impulse $I_{s p}$ and impulse coupling coefficient $C_{m}$. The specific impulse of classic propulsion systems is defined by [13]

$$
I_{s p}=\frac{F}{\dot{m} g}=\frac{p}{m g}=\frac{\langle v\rangle}{g} \quad\left[I_{s p}\right]=s,
$$

where $F$ is the thrust in Newton of the engine, $\dot{m}$ the mass flow rate of the propellant in $\mathrm{kg} / \mathrm{s}$ and $g$ the earth's standard acceleration on the surface measured in $\mathrm{m} / \mathrm{s}^{2} \cdot p$ is the impulse of the exhaust jet, in our case the ablated material and $\langle v\rangle$ the mass weighted velocity of $N$ expelled particles,

$$
\langle v\rangle=\frac{\sum_{i=1}^{N} m_{i} v_{i}}{\sum_{i=1}^{N} m_{i}} .
$$

The impulse coupling coefficient $c_{m}$ is defined by [15]

$$
c_{m}=\frac{\Delta \boldsymbol{p}}{E_{\text {laser }}}=\frac{\boldsymbol{p}_{\text {plume }}}{E_{\text {laser }}} \quad\left[c_{m}\right]=\frac{\mathrm{Ns}}{\mathrm{J}}=\frac{\mathrm{N}}{\mathrm{W}} .
$$

The ratio of the impulse change $\Delta p$ to the laser pulse energy $E$ is an important figure of merit of a propulsion system. Under the assumption of a non moving sample before ablation, the second part of the formula holds, which will mainly be used in the following.

\subsection{Hydrodynamic model - Virtual Laser Lab}

At the ITP, initial estimation parameters for MICROLAS propulsion were obtained by using Virtual Laser Lab (VLL). VLL is an online tool for onedimensional calculations of laser matter interactions, e.g. the behavior of thin metal foils has been simulated properly [17]. It is solving Maxwell's equations and a hydrodynamic continuum model in one dimension. Equations of state 
cover two main models, a Drude-like model valid for temperatures below $T_{\text {Fermi }}$ and a plasma model for the excited state above $T_{\text {Fermi }}$, both connected by a smooth transition. Also TTM equations are solved and connected to a single fluid material model. More details are given in [17].

\section{Results}

Table 1: Laser parameters of a recent laser ablation experiment at the DLR using a microchip laser

\begin{tabular}{|c|c|c|c|c|}
\hline Maximum energy & Wavelength & Spot diameter at the target & Fluence & Pulse duration \\
\hline$E_{\max }[\mu \mathrm{J}]$ & $\lambda[\mu \mathrm{m}]$ & $\omega_{0}[\mu \mathrm{m}]$ & $\Phi\left[\mathrm{J} / \mathrm{cm}^{2}\right]$ & $t_{\text {pulse }}[\mathrm{ps}]$ \\
\hline 80 & 1.064 & 30 & $3-10$ & 495 \\
\hline
\end{tabular}

Simulations were adjusted according to experimental values available in DLR laser laboratory. Main parameters of the system are given in table 1. For pulse width it follows that $\sigma_{t}=(2 \cdot \sqrt{2 \cdot \ln 2})^{-1} \cdot t_{P u l s e}=211 \mathrm{ps}$, so a width of 210 ps was chosen. According to the well known scaling law in laser ablation propulsion [14]

$$
C_{m} \sim\left(I \lambda \sqrt{t_{\text {Pulse }}}\right)^{-1 / 4}
$$

with $\lambda=$ const. and $I=\sigma_{e} / t_{P u l s e}, \sigma_{e}$ being the fluence, a law for same power input can be derived. For known $\sigma_{e, 1}$ and $t_{\mathrm{Pulse}, 1}$ the fluence for another pulse duration $t_{\mathrm{Pulse}, 2}$ follows from

$$
\sigma_{e, 2}=\frac{\sigma_{e, 1}}{\sqrt{t_{\mathrm{Pulse}, 1}}} \cdot \sqrt{t_{\mathrm{Pulse}, 2}}=\frac{\sigma_{e, 1}}{\sigma_{t, 1}} \cdot \sqrt{\sigma_{t, 2}}
$$

and thus for the chosen laser parameters

$$
\sigma_{e}=\frac{3 \mathrm{~J} / \mathrm{cm}^{2}}{\sqrt{210 \mathrm{ps}}} \cdot \sqrt{\sigma_{t}} .
$$

One main parameter set $\left(\sigma_{e}, \sigma_{t}\right)$ was simulated, i.e. $\left(9490 \mathrm{~J} / \mathrm{m}^{2}, 21 \mathrm{ps}\right)$. A simulation of the laser system of $\left(30000 \mathrm{~J} / \mathrm{m}^{2}, 210 \mathrm{ps}\right)$ as given in laboratory was out of reach due to the limits in computation time. In current implementation it is not possible to restart an interrupted TTM simulation from the last checkpoint. An interruption leads to configurations that are not in 
thermal equilibrium and thus will not give proper results when the simulation is restarted. The temperature of the ionic system would be the new starting temperature for both electronic and ionic subsystem, resulting in an artificial energy loss.

Table 2: Material parameters for heat conduction in TTM-model

\begin{tabular}{|c|c|c|c|}
\hline Material & $\begin{array}{c}\text { Electron heat } \\
\text { capacity coefficient } \\
\gamma\left[\frac{\mathrm{J}}{\mathrm{m}^{3} \mathrm{~K}^{2}}\right]\end{array}$ & $\begin{array}{c}\text { Electron thermal } \\
\text { conductivity } \\
K\left[\frac{\mathrm{J}}{\mathrm{Kms}}\right]\end{array}$ & $\begin{array}{c}\text { Electron-phonon- } \\
\text { coupling constant } \\
G\left[\frac{\mathrm{J}}{\mathrm{sm}^{3} \mathrm{~K}}\right]\end{array}$ \\
\hline Aluminum & $135^{1}$ & $235^{2}$ & $5.69 \cdot 10^{173}$ \\
\hline
\end{tabular}

${ }^{1}[12],{ }^{2}[3$, p. 759$],{ }^{3}[6]$

In table 2 the parameters used for TTM simulations are given. The Lambert-Beer penetration depth was set to $\mu=8 \mathrm{~nm}$ [23]. The simulated sample size in z-direction was $1.5 \mu \mathrm{m}$, in $\mathrm{x}$ - and $\mathrm{y}$ - direction $20 \mathrm{~nm}$ and included $37.5 \cdot 10^{6}$ aluminum particles. Crystal structures are fcc with a lattice constant of $4.0513 \AA$. As a first output, the density distribution over time is given in Fig. 2. The initial surface of aluminum bulk material lies at distance $0 \mathrm{~nm}$, the material is directed along the positive distance axis, negative values describe positions in vacuum in front of the aluminum sheet. On the time axis 0 ps defines the time of maximum intensity, at $3 \cdot \sigma_{t}=63 \mathrm{ps}$ most energy is coupled in. Material begins to melt at $-55 \mathrm{ps}$, evaporation starts at $-25 \mathrm{ps}$ and continues over the whole simulation time.

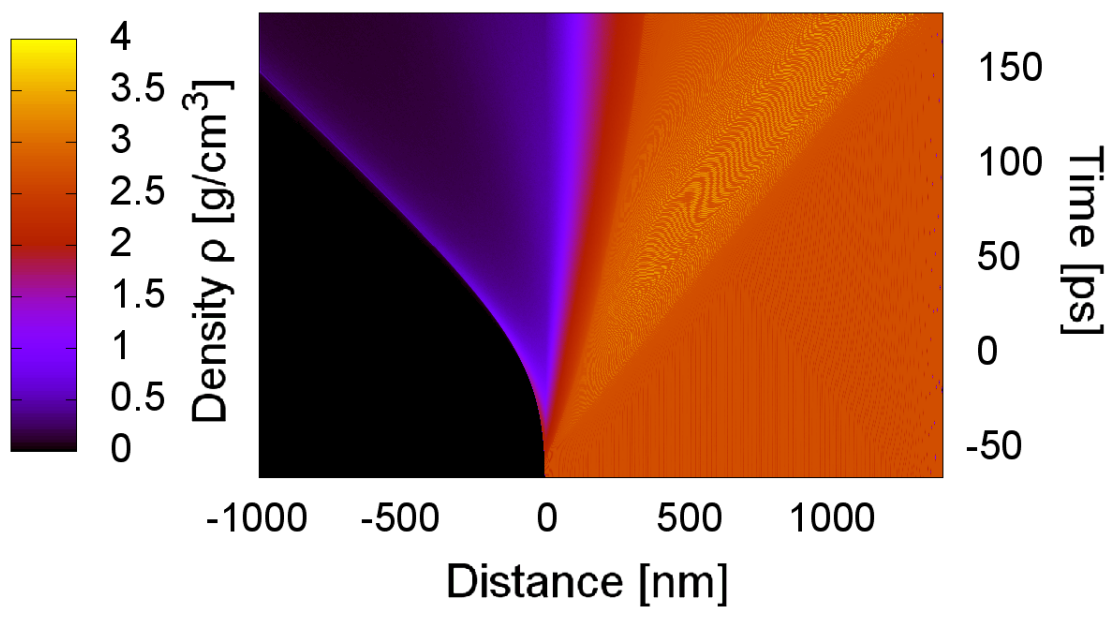

Fig. 2: Density plot for $\sigma_{t}=21 \mathrm{ps}, \sigma_{e}=9490 \mathrm{~J} / \mathrm{m}^{2}$ 
A pressure wave travels into the material with velocity

$$
\frac{1000 \mathrm{~nm}}{170 \mathrm{ps}} \approx 5.88 \frac{\mathrm{m}}{\mathrm{s}}
$$

which lies in the order of the velocity of sound waves in aluminum $\left(v_{\text {sound }} \approx 6.42 \mathrm{~km} / \mathrm{s}\right.$ at $\left.300 \mathrm{~K}[11, \mathrm{p} .14-36]\right)$. This region of compressed material is followed by a region of undistorted aluminum with normal solid density from $t=100 \mathrm{ps}$ on. At the surface of the material on the left a liquid layer develops. A vapor region follows, whereas a fluid mixture of both liquid and fluid separates both less dense regions. Temperature distributions of the electronic and ionic system vs. depth with respect to the initial surface at $0 \mathrm{~nm}$ are given in Fig. 5. The time is measured from the beginning of the simulation. In the first $200 \mathrm{fs}$ after simulation begins the lattice is still at $320 \mathrm{~K}$, while the interaction with the light field leads to an increase of electron temperature. It rises within the next $18 \mathrm{ps}$, the ionic system following due to electron-lattice relaxation. In Fig. 5 further time snapshots are given. Around 100 ps after starting the simulation, the current incoupling amounts to $\sim 17 \%$ of the maximum value, and the highest electronic temperature is achieved. Lattice and electronic system differ only by $15 \%$ at maximum at that time, with a decreasing difference to less than $4 \%$ after 215 ps simulation time. This configuration leads to runtimes which can be handled smoothly on multiple nodes at the High Performance Computing Center Stuttgart, so samples of the size used in this work can be simulated properly with irradiation of few ten picoseconds. Longer pulse durations results in non-equilibrated samples at the end of the simulation.
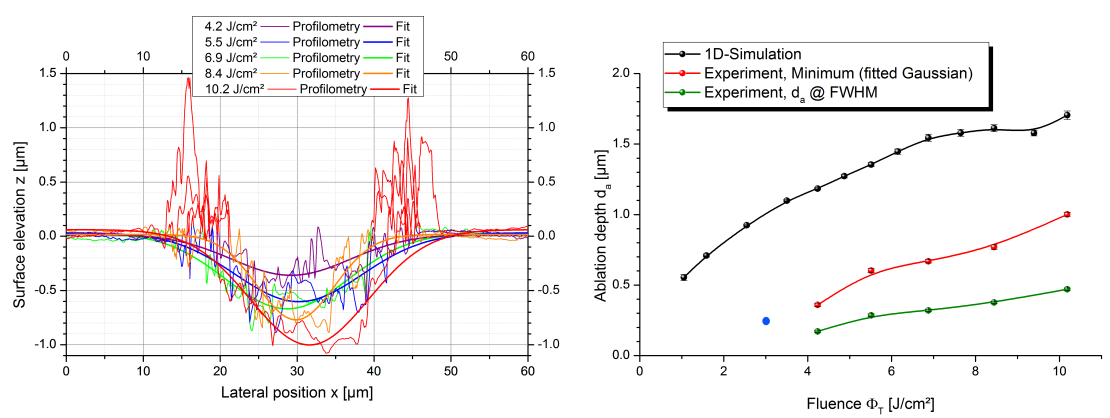

Fig. 3: An estimation of crater depths by Gaussian fits of experimental data (left) and in combination with VLL simulation output. The blue point refers to the IMD simulation of $\sigma_{t}=21$ ps (right) $[9,22]$.

Crater depths of ablation experiments can be compared to simulated depths, here namely melting depths. They are determined by finding the border position between solid and liquid phase in the density plots. White 
light interferometry of modified aluminum surfaces after laser pulse irradiation allows experimental access to crater depths. Typical experimental data of 210 ps ablation experiments in vacuum is given in Fig. 3 (left). A fit with Gaussian functions to the data points leads to full width half maximum depths as well as minimum depths. In Fig. 3 (right) average values over multiple craters are given. Additionally, liquid-solid interface position in the simulation of parameter set $\left(9490 \mathrm{~J} / \mathrm{m}^{2}, 21 \mathrm{ps}\right)$ are inserted for comparison. Under the pre-described assumptions they fit in quite well.

The checkpoints files given by IMD include particle masses, vectorial positions and velocities. The aerospace parameters specific impulse $I_{s p}$ and impulse coupling coefficient $C_{m}$ were calculated from these data via simple scripts. Fig. 6 shows their temporal behavior for the parameter pair (9490 $\mathrm{J} / \mathrm{m}^{2}, 21 \mathrm{ps}$ ). The way of choosing particles belonging to the ablated plume is quite naive, simply all particles with a spatial coordinate beyond a certain threshold are counted. In this case, all particles being $400 \mathrm{~nm}$ away from the initial surface were included. During simulation no recondensation processes could be depicted due to short simulation times since these processes happen on timescales of microseconds, which are not in reach at present. As shown in to Fig. 6 the parameters were least-squares fitted with two functions of the form

$$
\begin{aligned}
I_{s p} & =I_{1}+I_{2} \cdot e^{-a \cdot t} \\
C_{m} & =C_{1}+C_{2} \cdot\left(1-e^{-b \cdot t}\right),
\end{aligned}
$$

resulting in long time values for $t \rightarrow \infty$ given in table 3 . The same was done for hydrodynamic one-dimensional simulations with the code VLL [17], with simulation times in the nanosecond range. In the table the values are given for the second IMD simulation together with the data for 210 ps estimated with the experimental formula derived by Phipps et al.
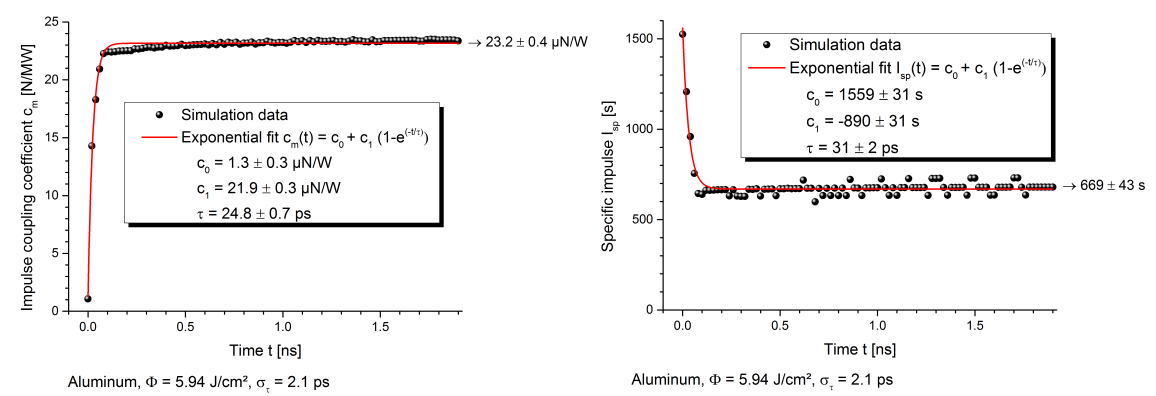

Fig. 4: Impulse coupling coefficient and specific impulse from VLL simulations, $\sigma_{t}=2.1 \mathrm{ps}$, $[22]$ 

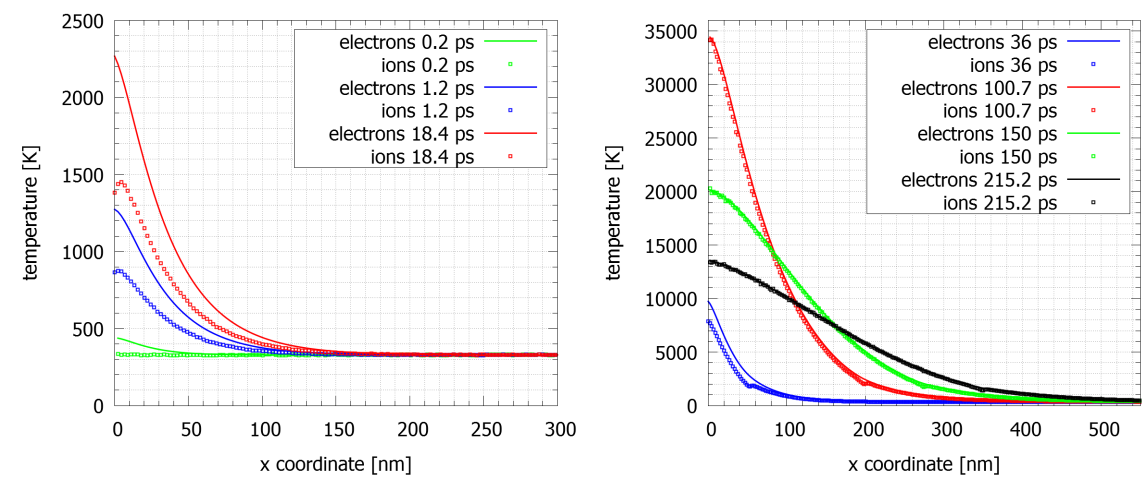

Fig. 5: Spatial distribution of electron and ion temperature. Left: at the beginning of the simulation. Right: towards the end of the simulation
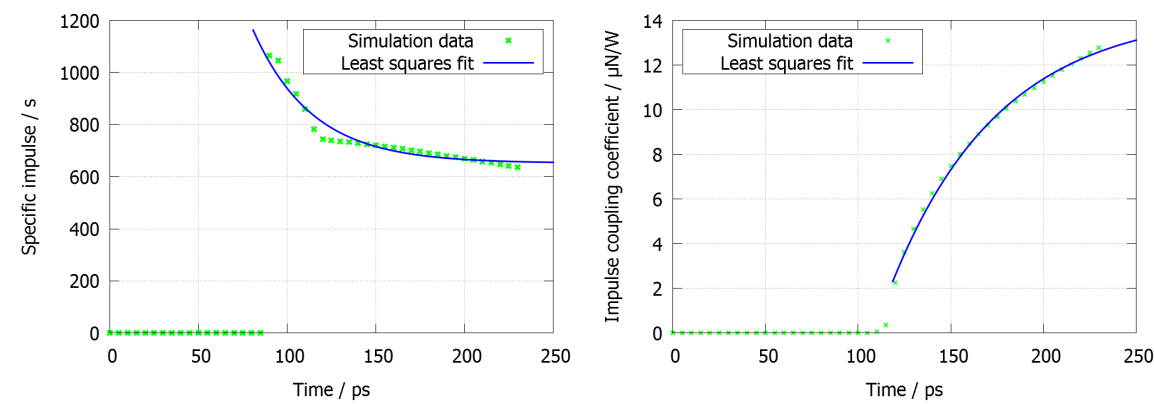

Fig. 6: Time evolutions. Left: of specific impulse. Right: of impulse coupling coefficient

In experiments with polymer and metal targets the Phipps formula was proven to estimate the aerospace parameters properly down to a lower limit of $t_{\text {pulse }}=100 \mathrm{ps}\left(\sigma_{t} \approx 42.47 \mathrm{ps}\right)[7]$.

The trend function formula for aluminum leads to an estimated value of [15]:

$$
\begin{aligned}
c_{m}[\mu \mathrm{N} / \mathrm{W}] & =\frac{55.6}{\left(\Phi \lambda \sqrt{t_{\mathrm{Pulse}}}\right)^{0.301}} \\
& =\frac{55.6}{\left(2.998 \mathrm{~J} / \mathrm{cm}^{2} \cdot 1.064 \mu \mathrm{m} \cdot \sqrt{500 \mathrm{ps}}\right)^{0.301}} \\
\Rightarrow c_{m} & \approx 25 \mu \mathrm{N} / \mathrm{W}
\end{aligned}
$$

VLL data seems to fit well although one order of magnitude lies between simulated and experimental pulse duration, and also between VLL and the IMD simulations performed. The results from the latter differ by a factor of 1.5-1.7 compared to VLL values. However, both aerospace parameters computed by IMD were not saturating within computation time and are likely to approach 
VLL findings. Phipps et al. and other groups did not yet publish findings about pulse durations around the simulated one, i.e. 21 ps. However, they investigated the few $100 \mathrm{fs}$ regime [16] for different materials. They found a slight decrease in $C_{m}$ compared to the 100 ps regime and state an optimal fluence for which they published $C_{m}$ and $I_{s p}$. Although optimal fluence is not directly comparable, the order of magnitude for both aerospace parameters remains the same as for longer pulses. The author's inference is that both will not differ much in the regime in between [16]. Experimental and modeling values are given in table 3 . Since no ultrashort pulse effects are included, a deviation in a factor of 2 from experimental findings is observed. Open questions arise from the simulations with IMD. Longer short pulse simulations are within reach and the future will show a more detailed behavior in this regime. Additionally, the estimation of the aerospace parameters has to be improved.

Table 3: Estimated values of aerospace parameters and experimental values; $130 \mathrm{fs}$ data from [16]

\begin{tabular}{|c|c|c|c|c|}
\hline simulation & $\begin{array}{c}C_{m} \\
{[\mu \mathrm{N} / \mathrm{W}]}\end{array}$ & $\begin{array}{c}\text { standard } \\
\text { deviation } \sigma\left(C_{m}\right) \\
{[\mu \mathrm{N} / \mathrm{W}]}\end{array}$ & $I_{s p}[\mathrm{~s}]$ & $\begin{array}{c}\text { standard } \\
\text { deviation } \sigma\left(I_{s p}\right) \\
{[\mathrm{s}]}\end{array}$ \\
\hline $21 \mathrm{ps}$ & 14.4 & 0.4 & 651.8 & 7.6 \\
$21 \mathrm{ps}, \mathrm{VLL}$ & 25.0 & 0.1 & 435 & 3 \\
210 ps, Phipps exper. & 24.98 & - & - & - \\
130 fs, Phipps model & 36 & - & - & - \\
130 fs, Phipps exper. & 18 & - & 2245 & - \\
\hline
\end{tabular}

\section{Performance and Benchmarks}

The general performance of IMD has been addressed in the basic papers on the implementation of IMD [25, 18], where benchmarks are also given. The performance on more recent supercomputer architectures and the performance in simulations of laser ablation especially has been addressed in previous HLRS reports $[21,20,19]$.

The simulation module for laser ablation was originally intended for fspulses only. The simulation of satellite propulsion uses pulses up to ns which leads to much longer simulation runs involving inhomogeneities generated by expansion of the probe and the ablated material. The result is a degradation of the performance.

Rough benchmark behaviours can be seen in figure 7 . The simulations were carried out on an Intel Xeon Processor X5670 (Nehalem-EP) with 12 threads 


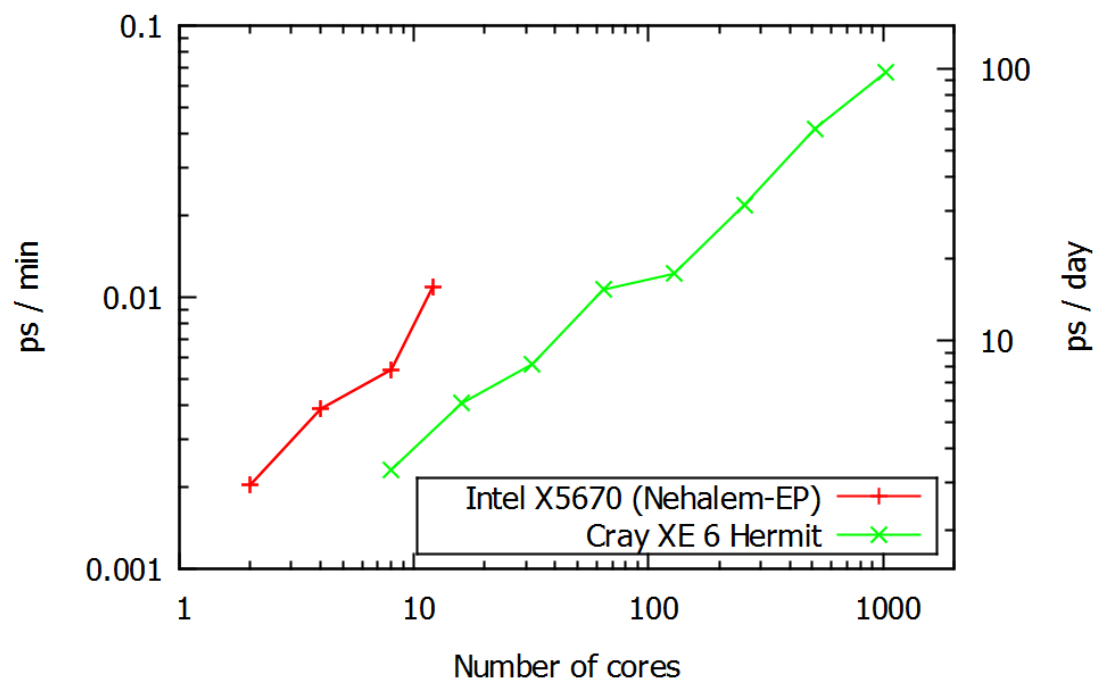

Fig. 7: Simulation time per real time minute for a NEC Nehalem compute node at ITAP and several nodes of the Cray System 'Hermit' at HLRS

(6 physical), running at $2.93 \mathrm{GHz}$ and one or several nodes on HLRS Hermit Cray XE 6 system, respectively. Each node has 32 threads (16 physical), being a Dual Socket AMD Interlagos CPU at $2.3 \mathrm{GHz}$. All simulations were carried out with a MD system of 12.5 million atoms. A linear scaling behaviour of cores to simulated time over real time in $\mathrm{ps} / \mathrm{min}$ can be infered. Although the scaling behaviour and total simulation time on the Nehalem CPU seems to surpass the multi-core system in first place, it reaches its maximum for 12 threads and is only expandable through interconnection of several nodes. Here the optimized system of HLRS plays off the standard simulation node. Still, there is a factor of 6 in total node number to achieve the same ratio of simulation to real time as Nehalem CPU. However, with increasing node number of course the absolute ratio also increases, leading to total simulation times of several 100 ps per day in the range of 1000 cores.

A general dynamical load balancing scheme has been implemented by Begau and Sutmann (High Performance Computing Center for Materials Science in Bochum) in IMD. In the case of a sphere colliding with a plate a performance gain of a factor of about two could be achieved for 64 to 256 CPUs. The data indicate that this gain will not decrease for higher CPU numbers. Since the colliding sphere setup is similar to inverse ablation it is likely that the load balancing can be applied to ablation also. Unfortunately, no benchmark data are currently available. The dynamical load balancing scheme of Begau and Sutmann will be adapted expecially to the simulations of laser ablation in the next period of the laser ablation subproject B.5 of SFB 716 . 


\section{Summary}

The fully parallelized open source package IMD was evaluated with respect to a new application in the aerospace field. Although underlying limitations and not direct comparability with experiments was given, it is suitable for calculations regarding MICROLAS propulsion. In near future investigations will be focused in first place on ultrashort laser pulses in the femtosecond regime to ensure direct comparability. For longer laser pulses absorptivity will play a main role, changing over time during irradiation. Long term planning will allow for three dimensional spatially resolved crater forming.

\section{Acknowledgment}

Financial funding from the German Science Foundation DFG for the Collaborative Research Center SFB 716 "Dynamic simulations of systems with large particle numbers" in subproject B.5 "Laser ablation: from simple metals to complex materials" is greatly acknowledged.

\section{References}

1. Workshop Mikroantriebe, 16./17.4.2013, Inst. f. tech. Physik, DLR Stuttgart, http: //www.dlr.de/tp/Portaldata/39/Resources//Agenda.pdf; visited at June 19, 2013.

2. M. Armano, M. Benedetti, J. Bogenstahl, and D. Bortoluzzi. LISA Pathfinder: the experiment and the route to LISA. Class. Quant. Grav., 26(9):094001, 2009.

3. D. Bäuerle. Laser processing and chemistry. Springer Berlin Heidelberg, 2011.

4. H. Dittus and T. van Zoest. Applications of microthrusters for satellite missions and formation flights scenarios. AIP Conf. Proc. 1402:367-373, 2011.

5. F. Ercolessi and J. B. Adams. Interatomic potentials from first-principles calculations: The force-matching method. Europhys Lett., 26:583, 1994.

6. B. Hüttner and G. Rohr. On the theory of ps and sub-ps laser pulse interaction with metals i. surface temperature. Appl. Surf. Sci., 103:269 - 274, 1996.

7. J. Ihlemann, F. Beinhorn, H. Schmidt, K. Luther, and J. Troe. Plasma and plume effects on UV laser ablation of polymers. Proc. SPIE 5448, High-Power Laser Ablation $V$, pp 572-580, 2004.

8. D. S. Ivanov and L. V. Zhigilei. Combined atomistic-continuum modeling of shortpulse laser melting and disintegration of metal films. Phys. Rev. B, 68:064114, 2003.

9. S. Karg and V. Fedotov. Investigation of laser-ablative micropropulsion as an alternative thruster concept for precise satellite attitude and orbit control. ONERA-DLR Aerospace Symposium 2013, Palaiseau, France, May 27-29, 2013.

10. S. Karg, S. Scharring, and H.-A. Eckel. Microthruster research activities at dlr stuttgart - status and perspective. AIP Conf. Proc. 1402:374-382, 2011.

11. D. Lide. CRC Handbook of Chemistry and Physics; 78th Ed. 1997-98. CRC Press, 1997.

12. Z. Lin, L. V. Zhigilei, and V. Celli. Electron-phonon coupling and electron heat capacity of metals under conditions of strong electron-phonon nonequilibrium. Phys. Rev. $B, 77,2008$. 
13. E. Messerschmidt and S. Fasoulas. Raumfahrtsysteme. Springer, fourth edition, 2011.

14. C. R. Phipps, M. Birkan, W. Bohn, H.-A. Eckel, H. Horisawa, T. Lippert, M. Michaelis et al. Review: laser-ablation propulsion. J. Prop. and Power, 26:609-637, 2010.

15. C. R. Phipps and J. R. Luke. Advantages of a ns-pulse micro-laser plasma thruster. AIP Conf. Proc. 664, pp 230-239, 2003.

16. C. R. Phipps, J. R. Luke, D. J. Funk, D. S. Moore, J. Glownia, and T. Lippert. Measurements of laser impulse coupling at 130 fs. Proc. SPIE 5448, High-Power Laser Ablation V, pp 1201-1209, 2004.

17. M. E. Povarnitsyn, N. E. Andreev, P. R. Levashov, K. V. Khishchenko, and O. N. Rosmej. Dynamics of thin metal foils irradiated by moderate-contrast high-intensity laser beams. Phys. Plasmas 19, 2012.

18. J. Roth, F. Gähler, and H.-R. Trebin. A molecular dynamics run with 5.180.116.000 particles. Int. J. Mod. Phys C, 11:317-322, 2000.

19. J. Roth, J. Karlin, M. Sartison, A. Krauß, and H.-R. Trebin. Molecular Dynamics Simulations of Laser Ablation in Metals: Parameter Dependence, Extended Models and Double Pulses, pp 105 - 117. in High Performance Computing in Science and Engineering '12, eds. W. E. Nagel, D. B. Kröner, M. M. Resch, Springer, Heidelberg, 2012 .

20. J. Roth, J. Karlin, C. Ulrich, and H.-R. Trebin. Laser Ablation of Aluminium: Drops and Voids, pp 93 - 104. in High Performance Computing in Science and Engineering '11, eds. W. E. Nagel, D. B. Kröner, M. M. Resch, Springer, Heidelberg, 2011.

21. J. Roth, C. Trichet, H.-R. Trebin, and S. Sonntag. Laser Ablation of Metals, pp 159 - 168. in High Performance Computing in Science and Engineering '10, eds. W. E. Nagel, D. B. Kröner, M. M. Resch, Springer, Heidelberg, 2010.

22. S. Scharring. Post-processing and visualization of simulation data which have been obtained through the use of virtual laser laboratory (http://vll.ihed.ras.ru), unpublished results (2013).

23. S. Sonntag. Computer simulations of Laser Ablation: From Simple Metals to Complex Metallic Alloys. PhD thesis, Universität Stuttgart, 2011.

24. S. Sonntag, J. Roth, and H.-R. Trebin. Molecular dynamics simulations of laser induced surface melting in orthorhombic $\mathrm{Al}_{13} \mathrm{Co}_{4}$. Appl. Phys. A, 101: 77-80, 2010.

25. J. Stadler, R. Mikulla, and H.-R. Trebin. IMD: A software package for molecular dynamics studies on parallel computers. Int. J. Mod. Phys C, 8:1131-1140, 1997.

26. C. Ulrich. Simulation der Laserablation an Metallen. Diploma thesis, Universität Stuttgart, 2007. 